\title{
Le bassin de l'Aude du Néolithique à l'âge du Fer
}

\section{Pascale Barthès}

Édition électronique
URL : http://journals.openedition.org/adlfi/11949

ISSN : 2114-0502

Éditeur

Ministère de la culture

Référence électronique

Pascale Barthès, "Le bassin de l'Aude du Néolithique à l'âge du Fer », ADLFI. Archéologie de la France Informations [En ligne], Languedoc-Roussillon, mis en ligne le 01 mars 2004, consulté le 19 avril 2019. URL : http://journals.openedition.org/adlfi/11949

Ce document a été généré automatiquement le 19 avril 2019

(c) Ministère de la Culture et de la Communication, CNRS 


\title{
Le bassin de l'Aude du Néolithique à l'âge du Fer
}

\author{
Pascale Barthès
}

\author{
Identifiant de l'opération archéologique : \\ Date de l'opération : 1991 - 1993 (PT) ; 1995 (FP) \\ Inventeur(s) : Barthès Pascale
}

1 Cette opération a été mise en place dans le cadre d'une action thématique programmée du CNRS (Grands projets d'Archéologie métropolitaine), dont les différents axes de travail étaient coordonnés par Jean Guilaine. Il s'agissait pour notre part de réfléchir sur les modes de peuplement de ce territoire - plaine alluviale de l'Aude et du Fresquel, reliefs calcaires du Minervois et du nord des Corbières, flanc sud de la Montagne noire - en relançant sur le terrain la recherche de sites et en établissant un répertoire bibliographique de toutes les occupations humaines (habitats et sépultures) connues à ce jour.

2 En ce qui concerne la recherche sur le terrain, une quarantaine de nouveaux points ont été mis en évidence. La recherche était fondée, dans un premier temps, sur l'inventaire des mobiliers archéologiques recueillis par les prospecteurs occasionnels de ce secteur, certains découverts grâce à cette opération. Dans un second temps, des prospections pédestres ponctuelles s'effectuaient soit pour confirmer les sites pressentis, soit pour préciser la nature, la datation ou l'extension d'un site déjà patent.

3 Au terme de l'opération, le répertoire rassemble six cent soixante-dix sites pour la période et le territoire considérés. Des cartes de répartition ont été dressées pour chacune des grandes phases chronologiques. L'analyse spatiale de ces données ayant été publiée de façon synthétique [(Barthès, Pascale ; Bocqueret, P. ; Rancoule, Guy. 1995.) et (Barthès, Pascale. 1994.)] nous indiquons, parmi les sites nouvellement acquis, ceux qui nous semblent apporter le plus à la connaissance actuelle. 


\section{Néolithique moyen}

4 L'habitat du Rec de Touzéry à Rieux-Minervois, est l'un des rares sites chasséens d'ampleur moyenne détecté en zone de plateau pouvant constituer un jalon entre les occupations de grottes et d'abris (Grotte Gazel, Abri de Font-Juvénal) et les grands établissements de plaine (Cavanac, Berriac). À la limite des communes de Bizanet et SaintAndré-de-Roquelongue, en bordure de la plaine narbonnaise, c'est précisément un nouveau grand établissement qui a pu être identifié à Gaussan-Proubigue, à partir de mobiliers répartis sur une surface d'au moins 20 ha. L'intérêt de ce site est d'avoir livré des haches polies en cinérite originaire du Rouergue et de grandes haches en silex provenant du Bergeracois.

\section{Néolithique final/Chalcolithique}

Une occupation du Campaniforme régional détectée au Pradalas à Villeneuve-Minervois a donné lieu à une fouille de contrôle dirigée par Frédéric Jallet en 1995. Sur les sites de Robert à Douzens, de Foun de Madamo à Conilhac-Corbières, et du Pesquié à SaintLaurent-de-la-Cabrerisse, ont été découverts des déchets de coquilles en abondance et des perles discoïdes à l'état d'ébauche, témoins d'une activité de fabrication associée dans les deux premiers cas à un habitat (Fig. $n^{\circ} 1$ : Habitat du Néolithique final/Chalcolithique avec atelier de production de perles discoïdales sur cardium. 6 à $8:$ perles à l'état d'ébauche ; 10 et 11 : perles achevées; 4 et $12:$ pièces en silex de type Salinelles; 9 et $13:$ pendeloques sur test de coquillage roulé). Ces trois sites se trouvent tous à plus d'une trentaine de kilomètres de la côte. Ils posent pour la première fois en Midi méditerranéen la question de la production de ces parures particulièrement répandues dans les dolmens languedociens de la fin du Néolithique.

\section{Âge du Bronze}

6 Une partie du mobilier que recélait l'ossuaire de Mountpénéry à Boutenac, victime de fouille sauvage, a pu être étudié. Dans ce petit ensemble cohérent, attribuable à une phase ancienne de l'âge du Bronze, figure la partie supérieure d'un vase portant un décor complexe à base de triples lignes d'impression arciformes, type de décor qui ne trouve pas de parallèle parmi la céramique connue de cette période. Le site d'Al Bosc à Trausse, attribuable également au Bronze ancien, mérite une attention particulière en raison du faible nombre d'habitats de plein air recensés jusqu'ici sur ce territoire. Pour la fin de l'âge du Bronze, Pech Casti à Villeneuve-Minervois et Le Capitaine à Bagnoles sont probablement de petites occupations liées à la mise en valeur du plateau calcaire.

\section{Âge du Fer}

7 L'apport le plus significatif est constitué par le petit habitat de La Trille à Trausse, occupé durant le second âge du Fer (céramique à décor ondé, bracelet en verre bleu de tradition celtique) et perpétuant une installation du Néolithique final et de l'âge du Bronze final. 


\section{BIBLIOGRAPHIE}

Barthès, PascaleBocqueret, P.Rancoule, Guy. 1995 : « Prospection-inventaire dans le bassin de l'Aude entre Carcassonne et Narbonne ", in Guilaine et al., Temps et Espace dans le bassin de l'Aude du Néolithique à l'âge du Fer, Toulouse, Centre Anthropologie, p. 93-164.

Barthès, Pascale. 1994 : «Les premiers agriculteurs ", in Guilaine et al., Aube des Origines. Archéologie en terre d'Aude, Carcassonne, Groupe audois d'archéologie préhistorique, p. 67-72.

\section{ANNEXES}

Fig. $\mathrm{n}^{\circ} 1$ : Habitat du Néolithique final/Chalcolithique avec atelier de production de perles discoïdales sur cardium. 6 à 8 : perles à l'état d'ébauche; 10 et 11 : perles achevées; 4 et 12 : pièces en silex de type Salinelles; 9 et 13 : pendeloques sur test de coquillage roulé
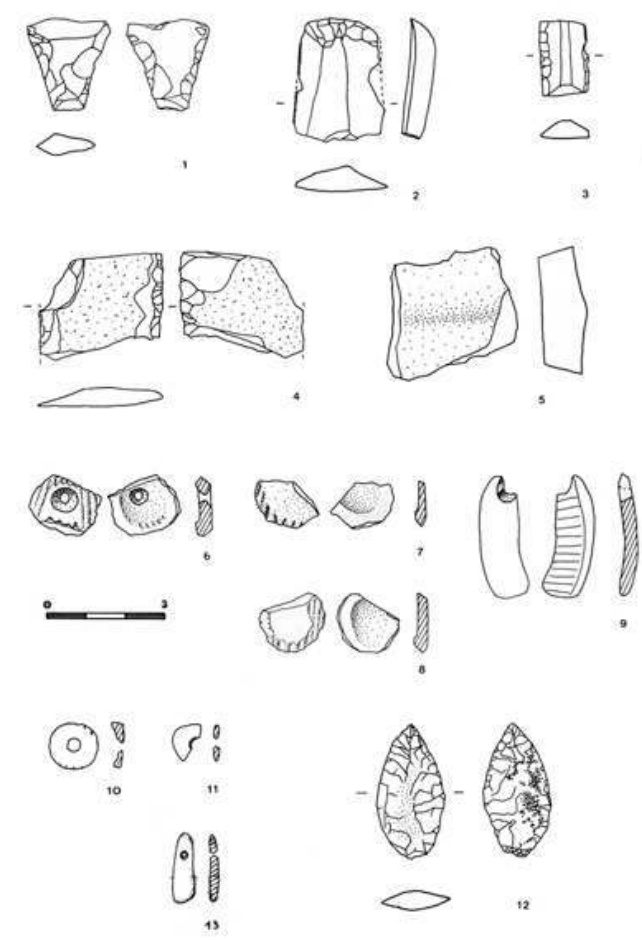

Auteur(s) : Douzens, Robert. Crédits : ADLFI - Douzens, Robert (2003) 
INDEX

operation Prospection thématique (PRT)

Index géographique : Languedoc-Roussillon, Aude (11), Trausse, Rieux-Minervois, VilleneuveMinervois, Douzens, Conilhac-Corbières, Bizanet, Saint-André-de-Roquelongue, Saint-Laurentde-la-Cabrerisse, Bagnoles, Boutenac

Index chronologique : âge du Bronze, âge du Fer, Néolithique, Néolithique final, Chalcolithique 